\title{
On Further Polygonal Designs using Linked Triangular Structures other than Spidrons and Tiling Patterns generated by them
}

\author{
T. Gangopadhyay \\ $\mathrm{XLRI}$ \\ C.H.Area(E), Jamshedpur, \\ India
}

\begin{abstract}
A regular $\mathrm{n}$-sided polygon can be split into $\mathrm{n} n$-part spidrons. Alternate forms of linked triangular structures such as ladders and creepers can also be used to subdivide regular polygons. In the present paper new symmetric designs with inscribed regular polygons are constructed using n 6-part ladders. Also several new tiling patterns are created using these designs
\end{abstract}

\section{General Terms}

Tiling, Algorithm, Turbo C++, Program.

\section{Keywords}

Spidron, ladder, polygon, isosceles, subdivision.

\section{INTRODUCTION}

An n-part spidron is a figure in plane geometry consisting entirely of an alternating sequence of two isosceles triangles, each with its own base angles[2], [7], [9]. The two triangles are juxtaposed in such a way that together they from a third bigger triangle. The sequence is then drawn at an appropriate angle on a smaller scale and the process is repeated ad infinitum. $n$-part spidrons have been largely studied for $n=6$ and $8[6]$. A regular $\mathrm{n}$-sided polygon can be split into $\mathrm{n} n$-part spidrons[8]. An 8-part spidron is displayed in Figure 1. Erdely[2], has called the same structure a semispidron.

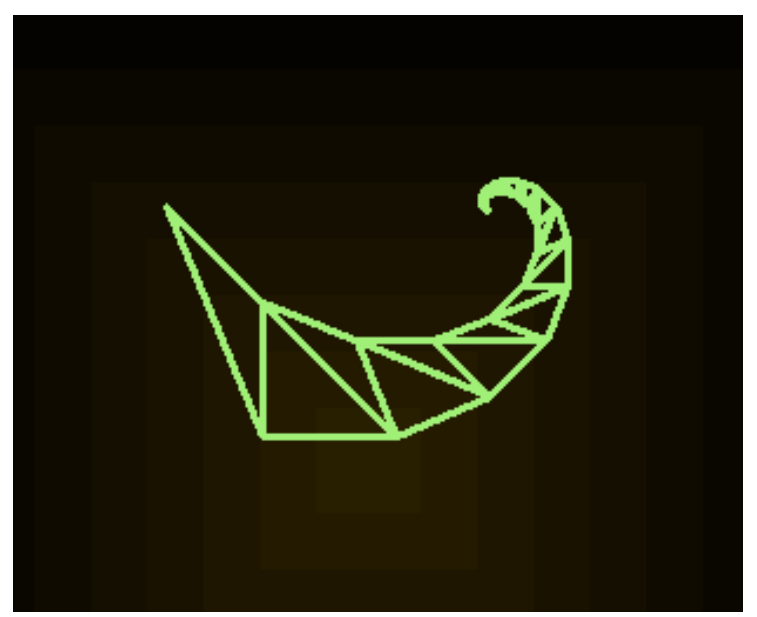

Fig 1 : An eight-part spidron

In earlier papers, the present author has developed an alternate way of constructing individual n-part spidrons so as to algorithmically juxtaposing them to produce a regular n-sided polygon (Gangopadhyay([3]) and has also shown that there exist other linked triangular structures such as ladders(Figure 2) and creepers(Figure 3) which are distinct from spidrons and which can also be used to subdivide regular polygons(Gangopadhyay([4],[5]).
In the present paper, the author presents new symmetric designs which inscribe regular polygons using $\mathrm{n}$ 6-part ladders and explores several tiling designs with the same. Sometimes two symmetric designs are superimposed to create the basic building block for a tiling pattern. These are primarily the distinctive features of this paper.

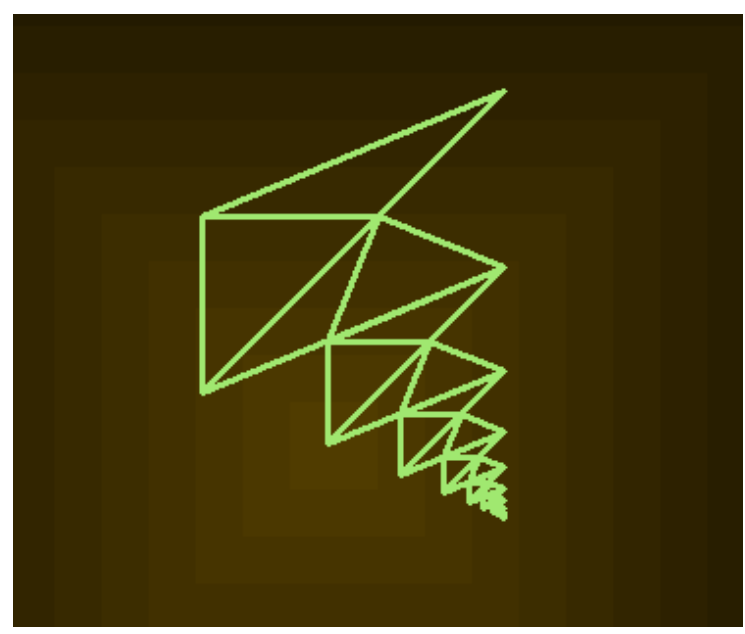

Fig 2 : An eight-part ladder

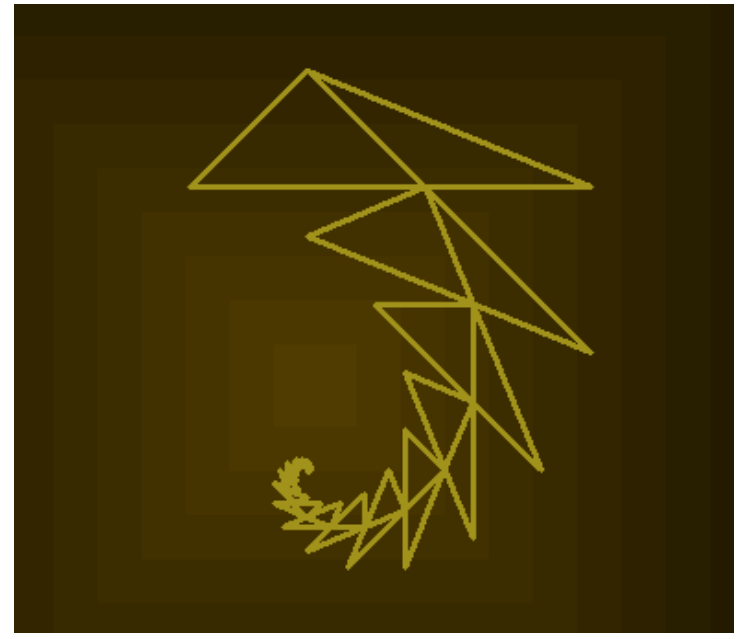

Fig 3 : An eight-part creeper

\section{THE ALGORITHM}

For the basic building block one uses two symmetric designs both created using six 6-part ladders. To do this one draws all ladders projecting outwards for the first design and then projecting inwards for the second design. These two designs are depicted in Figures 4 and 5 respectively. We also produce 
a third symmetric design by superimposing the first two. This is depicted in figure 6 . It is to be noted that all three designs have an inscribed hexagon.

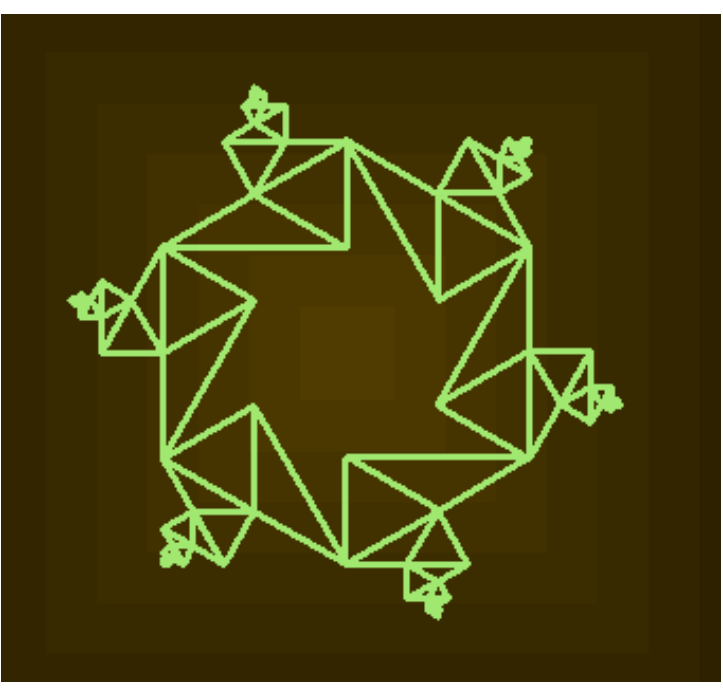

Fig 4 : The first symmetric design

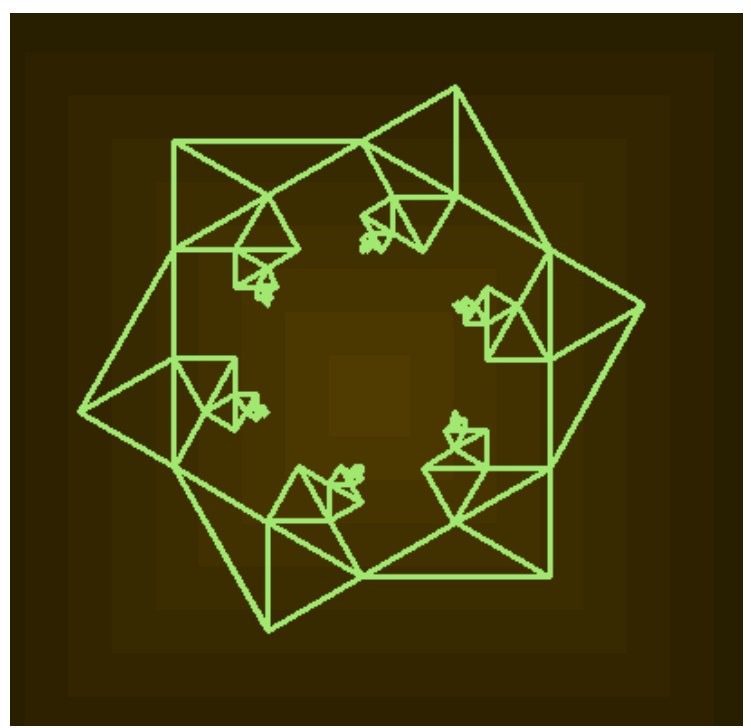

Fig 5 : The second symmetric design

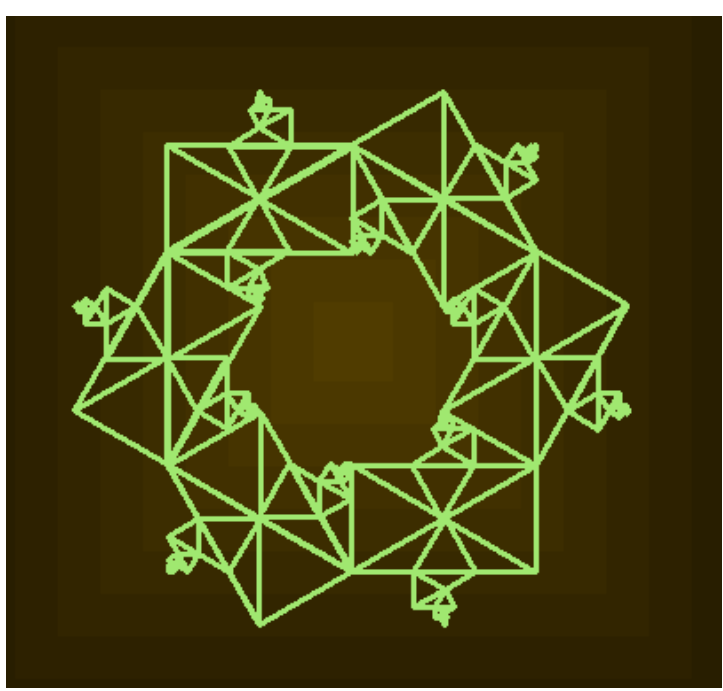

Fig 6 : The superimposed design
In the next section one submits a programming code in Visual $\mathrm{C}++$ that captures the algorithm and generates figures 4,5 and 6.

\section{THE CODE}

The code uses a function ladder which has two parameters $-\mathrm{s}$, which gives the value of the two equal sides of the first isosceles triangle and 1 , which specifies the number of recursive iterations. Both functions draw a single n-part ladder. Two other functions, polyladder1 and polyladder2 use the function ladder in a loop. Both polyladder1 and polyladder 2 use the function ladder, a forward move, a right rotation, another forward move and a left rotation, the only difference being that the right rotation is of $90+360 / \mathrm{n}$ for polyladder 1 and $90-360 / \mathrm{n}$ for polyladder 2 . The outputs of polyladder 1 and polyladder 2 are displayed in figures 4 and 5 for $n=6$. In the main body of the code, we call both polyladder1 and polyladder2 in that order while suitably changing the cursor. The result is shown in Figure 6. The functions $\mathrm{fd}, \mathrm{rt}, \mathrm{lt}$, pu and pd are adapted from turtle graphics[1] and have their usual connotations. The function $\mathrm{fd}$ draws a line of specified length, $\mathrm{rt}$ and lt respectively rotates the pen right and left by a specified angle, pu puts the pen up(no drawing) and pd puts the pen down. The various functions and the code are given below:

float ang $=90, p x, p y, p s=1$;

void fd(float dist)

\{float $\mathrm{hx}=\cos ($ ang $* 3.1415926536 / 180)$;

float hy $=\sin ($ ang $* 3.1415926536 / 180)$;

float $\mathrm{nx}=\mathrm{px}-\mathrm{hx} *$ dist;

float ny=py-hy*dist;

if(ps!=1)goto label;

line(px,py,nx,ny);

label:px=nx;py=ny; \}

float rt(float $\mathrm{l})$

$\{$ ang+=1;

return ang; \}

float lt(float 1$)$

\{ang-=1;

return ang; \}

void $\mathrm{pu}()$

$\{\mathrm{ps}=0 ;\}$

void $\mathrm{pd}()$

$\{\mathrm{ps}=1 ;\}$

int $n=6$; float ag=360/6; float ag1=360/n;

void ladder(float s,int 1 )

$\{$ if $(\mathrm{l}==0)\{\mathrm{fd}(\mathrm{s}) ; \mathrm{lt}(180-\mathrm{ag}) ; \mathrm{fd}(\mathrm{s}) ; \mathrm{fd}(2 * \mathrm{~s} * \cos (\mathrm{ag} * 3.14 / 180))$; lt(180-ag/2); fd( $\left.2 * \mathrm{~s}^{*} \cos (\mathrm{ag} / 2 * 3.14 / 180)\right) ; \operatorname{lt}(180-\mathrm{ag} / 2)$; $\mathrm{fd}(\mathrm{s}) ; \mathrm{rt}(180-\mathrm{ag}) ; \mathrm{pu}() ; \mathrm{fd}(\mathrm{s} * 2 * \cos (\mathrm{ag} * 3.14 / 180)) ; \mathrm{pd}() ;$ lt $(180-\mathrm{ag} / 2) ; \mathrm{fd}(\mathrm{s} * \cos (\mathrm{ag} * 3.14 / 180) / \cos (\mathrm{ag} / 2 * 3.14 / 180)+$ $2 * \mathrm{~s} * \cos (\mathrm{ag} * 3.14 / 180) * \cos (\mathrm{ag} * 3.14 / 180) / \cos (\mathrm{ag} / 2 * 3.14 / 180))$; lt(180-ag);

$\mathrm{fd}(\mathrm{s} * \cos (\mathrm{ag} * 3.14 / 180) / \cos (\mathrm{ag} / 2 * 3.14 / 180)) ; \mathrm{lt}(2 * \mathrm{ag})$; $\mathrm{fd}(\mathrm{s} * \cos (\mathrm{ag} * 3.14 / 180) / \cos (\mathrm{ag} / 2 * 3.14 / 180)) ; \mathrm{tt}(\mathrm{ag} / 2)$; return;\}

ladder(s,0);

ladder $((\mathrm{s}) * \cos (\mathrm{ag} * 3.14 / 180) *$ $\cos (\mathrm{ag} * 3.14 / 180) /(\cos (\mathrm{ag} / 2 * 3.14 / 180) * \cos (\mathrm{ag} / 2 * 3.14 / 180))$, 
$1-1) ;\}$

void polyladder1(float $\mathrm{s}$, int $\mathrm{l}$ )

$\{$ for(int $\mathrm{i}=0 ; \mathrm{i}<\mathrm{n} ; \mathrm{i}++)\{$

float $\mathrm{a}=\mathrm{px}, \mathrm{b}=\mathrm{py}$;

ladder(s,l);px=a,py=b;fd(s);pu();rt(90+ag1);

$\mathrm{fd}(2 * \mathrm{~s} * \cos (\mathrm{ag} / 2 * 3.14 / 180)) ; \operatorname{lt}(90) ; \operatorname{pd}() ;\}$

\}

void polyladder2(float $\mathrm{s}$, int $\mathrm{l}$ )

$\{$ for $($ int $\mathrm{i}=0 ; \mathrm{i}<\mathrm{n} ; \mathrm{i}++)\}$

float $\mathrm{a}=\mathrm{px}, \mathrm{b}=\mathrm{py}$;

ladder(s,1);px=a,py=b;fd(s);rt(90-ag1);

$\mathrm{fd}(2 * \mathrm{~s} * \cos (\mathrm{ag} / 2 * 3.14 / 180)) ; ; \operatorname{lt}(90) ; \operatorname{pd}() ;\}$ \}

void main()

$\{$ initwindow $(1000,800)$;

setlinestyle(SOLID_LINE,0,THICK_WIDTH);

float $\mathrm{s}=60$;

$\mathrm{px}=500, \mathrm{py}=350$

polycreeper $1(\mathrm{~s}, 15)$;

$\mathrm{px}=603, \mathrm{py}=530$;

polycreeper $2(\mathrm{~s}, 15)$;

getch();

closegraph();

\}

The output of the sample code is illustrated in Figure 7 where $\mathrm{n}$ has been set to 8 and the cursor has been appropriately changed - resulting in a superimposed design inscribing a regular octagon.

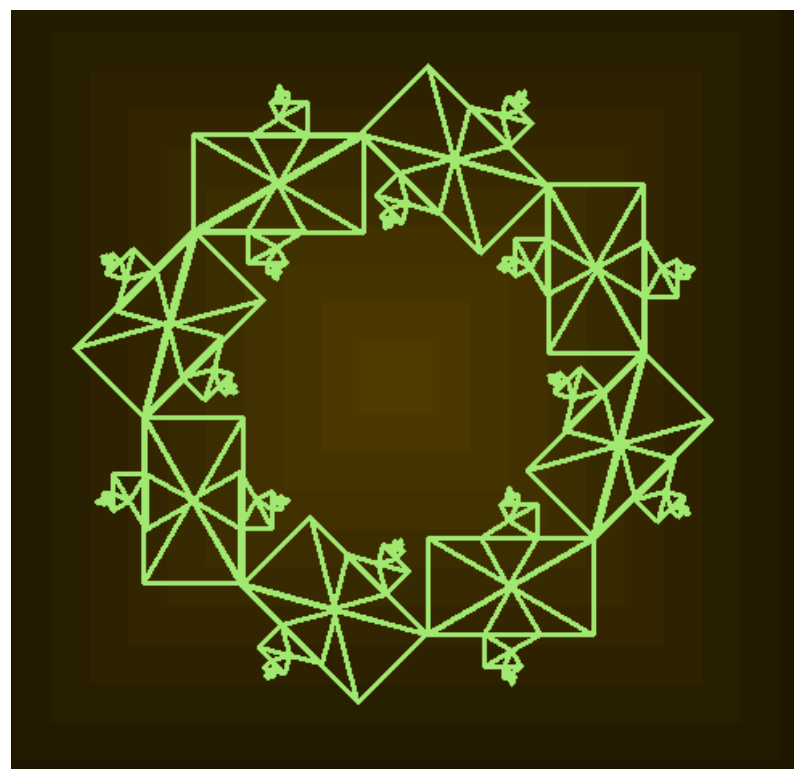

Fig 7: Output of the sample code, $n=8$

Figures 8 and 9 depict the respective outputs for $n=5$ and $n=4$ with appropriate changes for the cursor. For figure $8(n=5)$, we also need a left rotation by 36 degrees between the two calls of polyladder 1 and polyladder2.

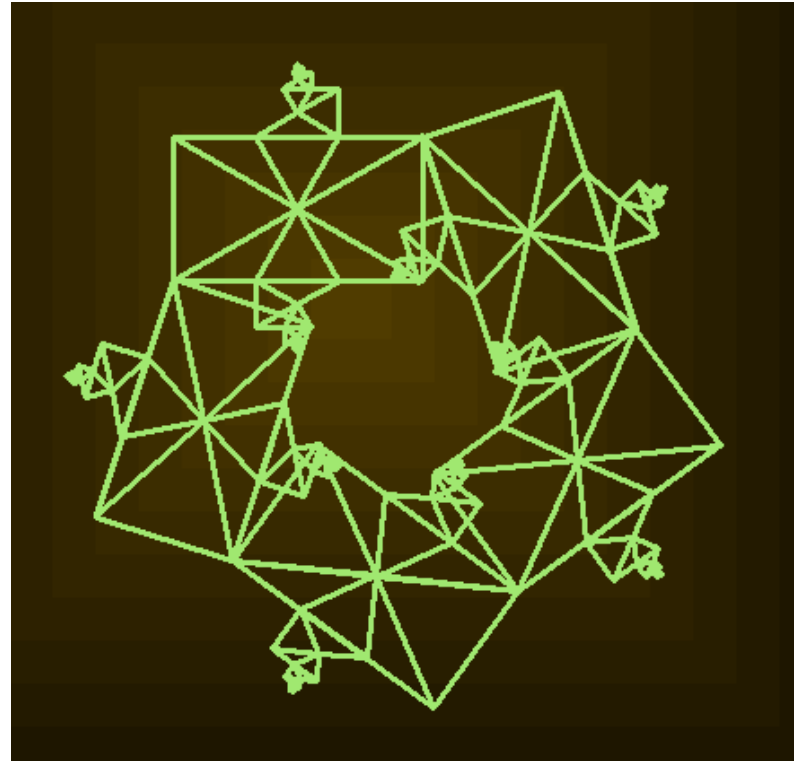

Fig 8: Output of the sample code, $n=5$

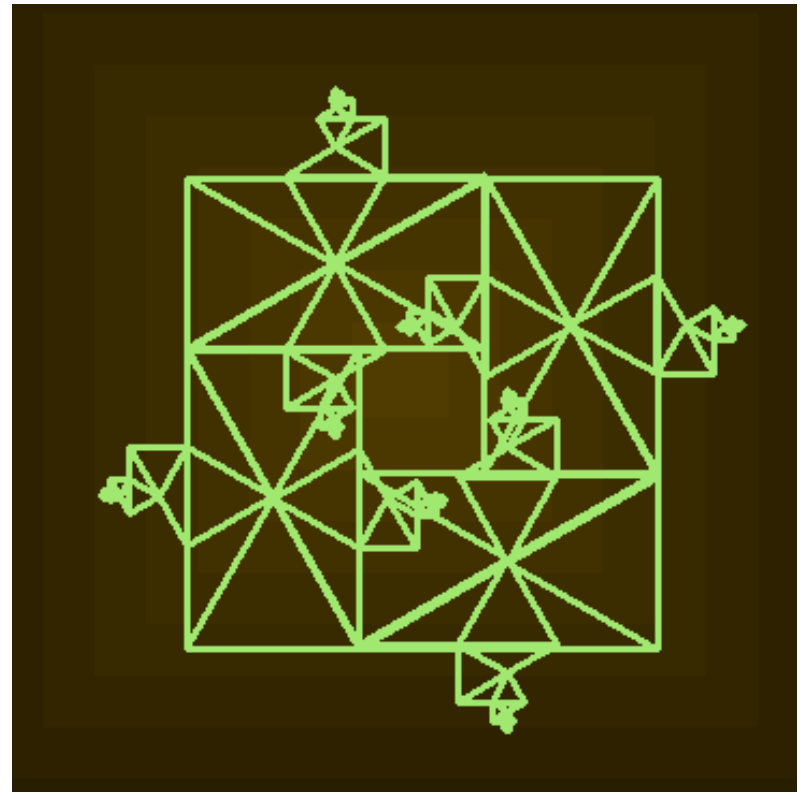

Fig. 9 : Output of the sample code for $n=4$

\section{TILING THE PLANE USING THE POLYGONAL DESIGNS}

Tilings with the first symmetric design are displayed in figures 10,11 and 12 respectively for $n=4,6$ and 8 . Tilings with the second symmetric design are displayed in figures 13,14 and 15 respectively for $n=4,8$ and 12 . Finally, figures 16 and 17 respectively show the effect of the superimposed design on tiling patterns for $n=8$ and 6 , while figure 18 show the effect of using both symmetric designs separately to generate a tiling pattern for $\mathrm{n}=3$. 


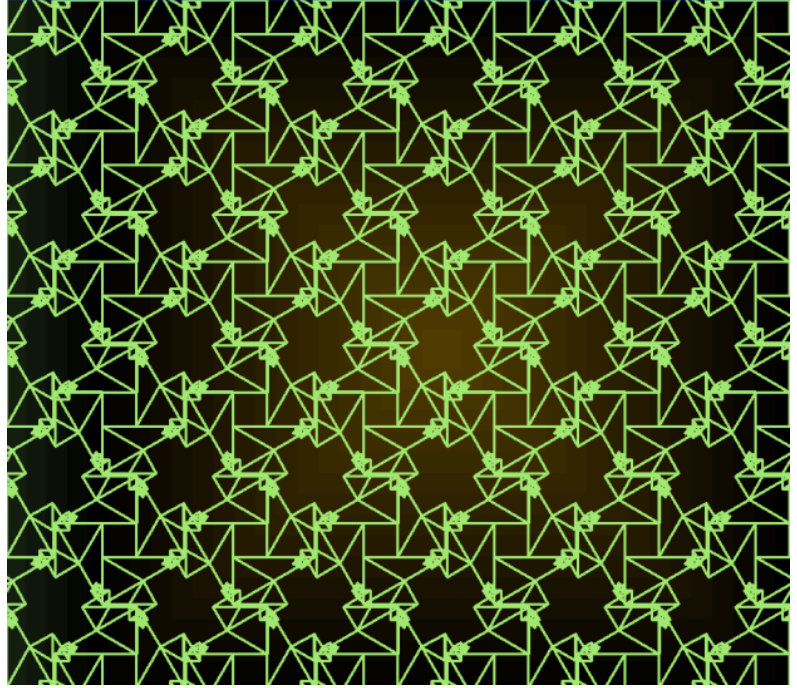

Fig. 10: Tiling pattern with the first symmetric design, $n=4$

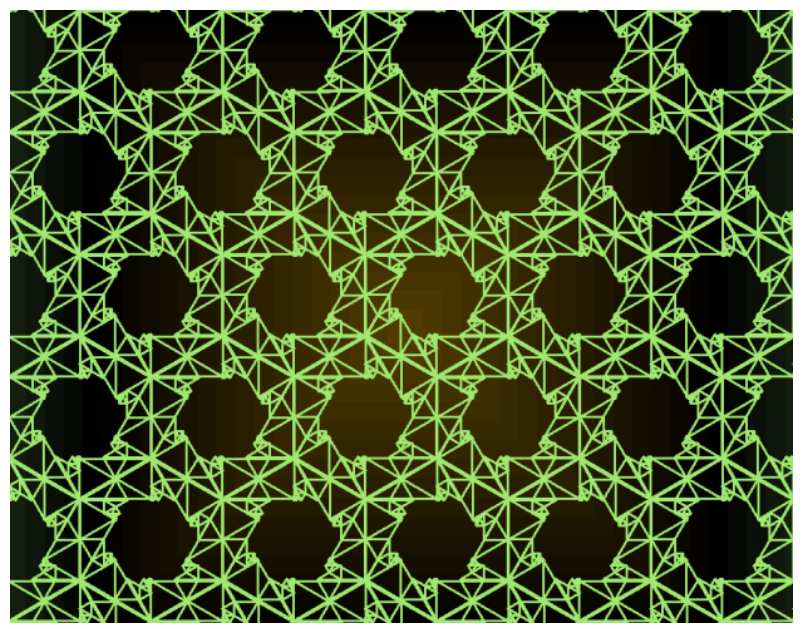

Fig. 11: Tiling pattern with the first symmetric design, $n=6$

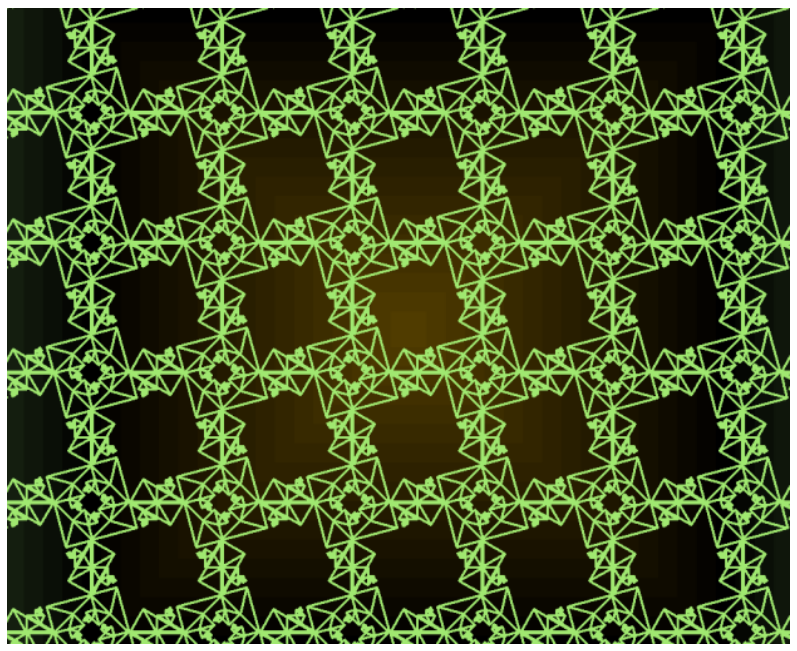

Fig. 12: Tiling pattern with the first symmetric design, $n=8$

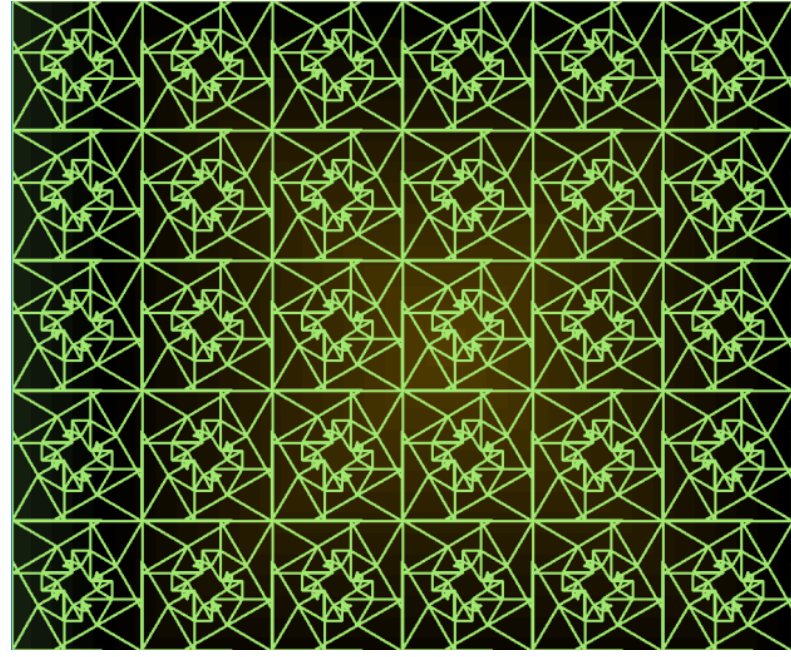

Fig.13: Tiling pattern with the second symmetric design, $n=4$

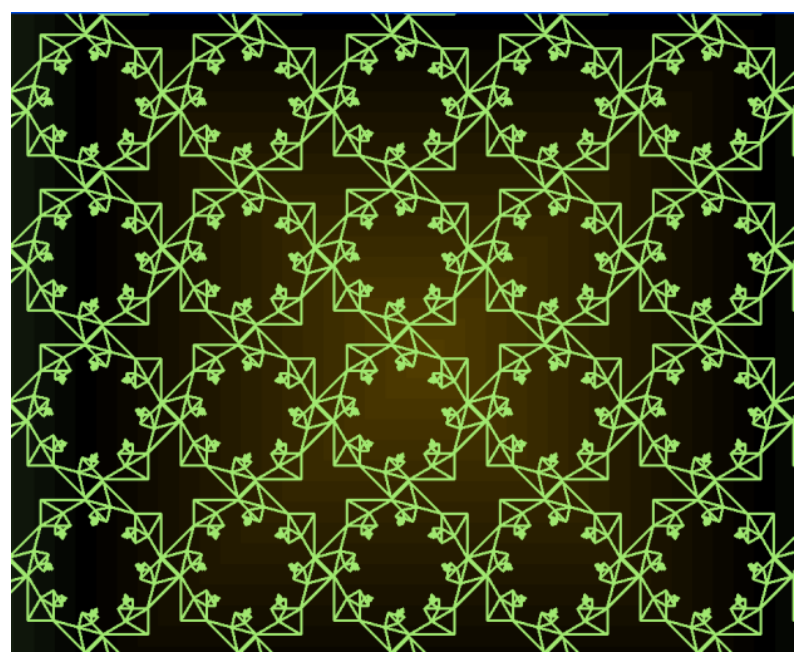

Fig.14: Tiling pattern with the second symmetric design, $\mathrm{n}=\mathbf{8}$

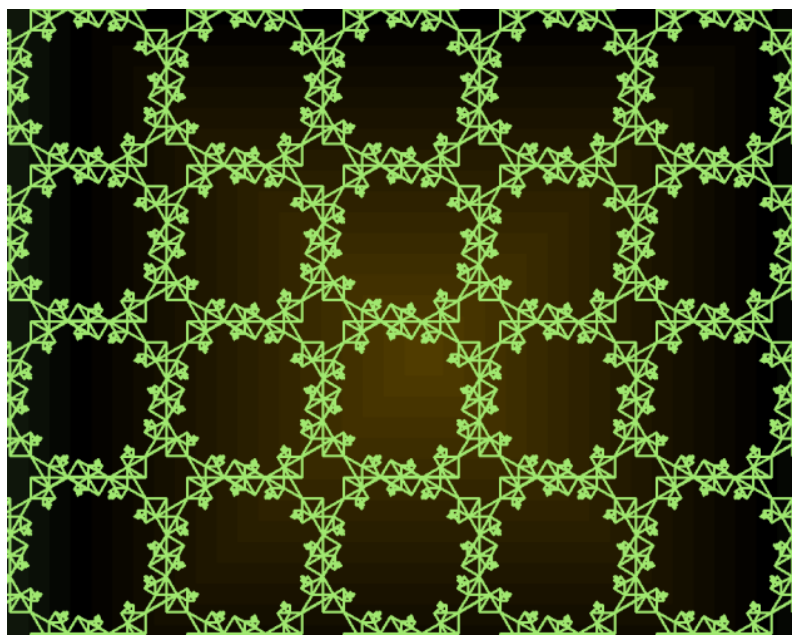

Fig.15: Tiling pattern with the second symmetric design, $\mathrm{n}=12$ 


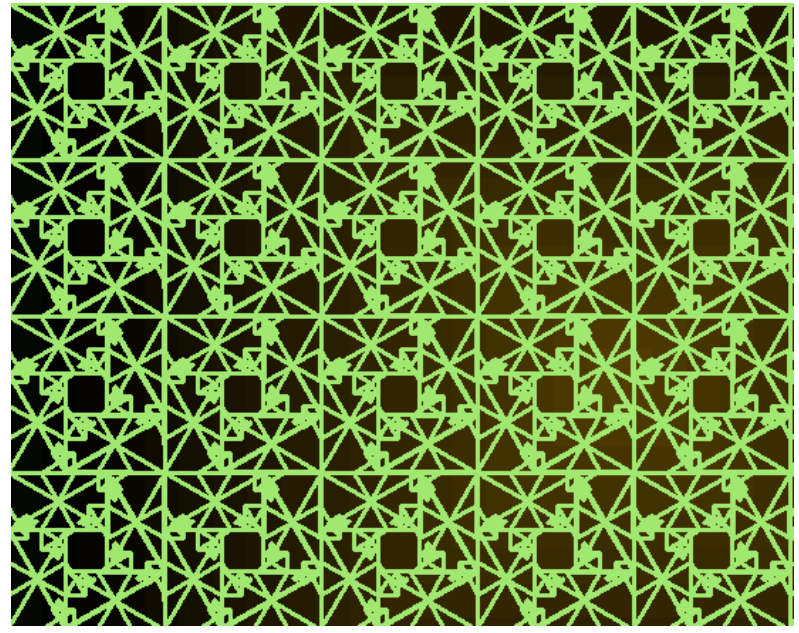

Fig.16: Tiling pattern with the superimposed design, $n=4$

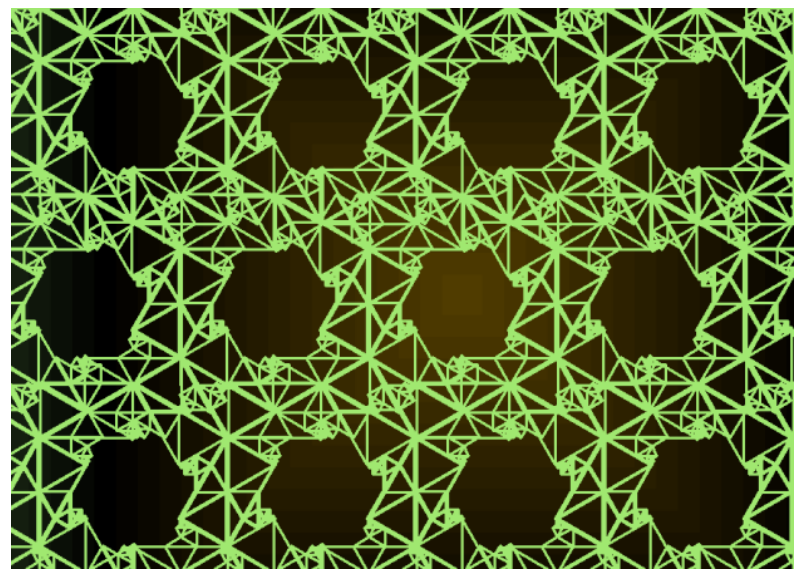

Fig.17: Tiling pattern with the superimposed design, $n=6$

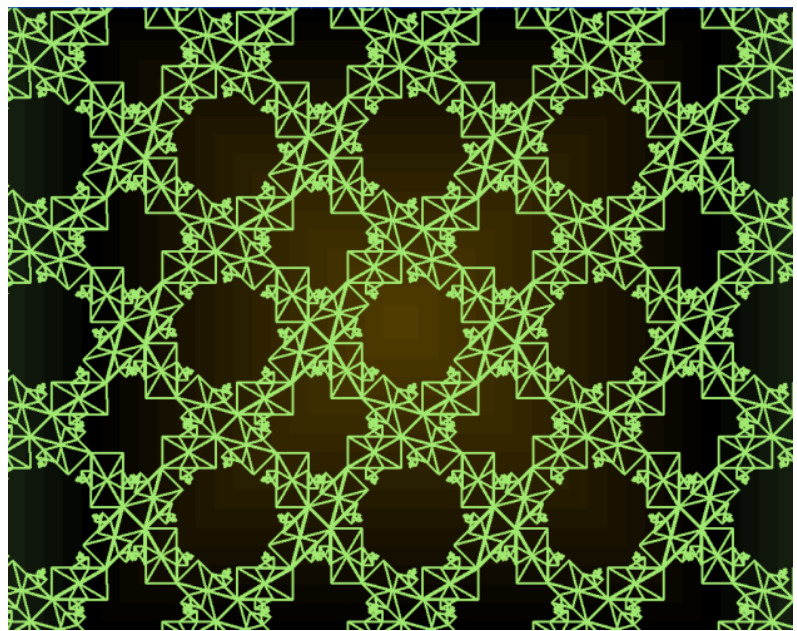

Fig.18: Tiling pattern with the superimposed design, $n=8$

\section{CONCLUSION}

This paper presents new symmetric designs which inscribe regular polygons using $\mathrm{n}$ 6-part ladders and explores several tiling designs with the same.. In subsequent studies one will further explore aperiodic tiling patterns created with polygonal designs using an odd number of ladders. Further, the effect of linked triangular structures other than spidrons ladders and creepers on tiling patterns will also be studied. These are the aspects that would be explored in future work.

\section{ACKNOWLEDGMENTS}

The author wishes to acknowledge his debt to the referee(s) for their constructive suggestions and encouragement

\section{REFERENCES}

[1] Abelson and diSessa, Turtle Geometry, MIT Press, 1992

[2] Erdely, D. Bridges 2007 Exhibit http://www.bridgesmathart.org/artexhibits/bridges2007/erdely.html.

[3] Gangopadhyay, T. On an alternate construction method for generating spidrons and new tiling patterns generated by them, International journal of Computer Applications, Volume 160, number 3, 2017.

[4] Gangopadhyay, T. On subdividing regular polygons using structures other than spidrons and tiling patterns generated by them, International journal of Computer Applications, volume163, number2, 2017

[5] Gangopadhyay, T. On further subdivisions of regular polygons using structures other than spidrons and tiling patterns generated by them, International journal of Computer Applications, volume165, number1, 2017

[6] Jacques, F. Spidrons d'octogone http://polyspidrons.overblog.com/article-4823990.html

[7] Peterson, I. "Swirling Seas, Crystal Balls". ScienceNews.org. Archived from the original on February 28, 2007. Retrieved 2007-02-14.

[8] Stenzhorn, S. Mathematical description of Spidrons, http://stefanstenzhorn.com/Spidrons.

[9] Spidron: https://en.wikipedia.org/wiki/Spidron. 